\title{
The Power Supplies Demand Conditions of the Big Data Technology Optimization Model
}

\author{
Fei Shi, Yonghuan Hu and Fengna Dong \\ 1122 YuanShen road, Shanghai, China
}

\begin{abstract}
Power supplies timely supply for the stable operation of the national grid and construction has the vital role. In this article, through the analysis of the characteristics of the electricity supplies demand, from the perspective of demand side, the production side, supply side research the contradiction between supply and demand of power supplies, so it is concluded that current power supplies demand patterns are faced with the practical problems, and gives the power supplies are optimized by the big data technology demand patterns theory method.
\end{abstract}

Keywords-power supplies management; characteristics of supply and demand; big data technology; model optimization

\section{INTRODUCTION}

With the rapid development of digital information era, based on Internet technology application and development of "big data" is a positive impact on all walks of life. In the power industry, the rapid development of the smart grid makes the grid business data show explosive growth, big data "quantity, class," the characteristics of has further highlights in the business of power networks, power big data analysis is urgently needed.

Before with the gradual improvement of power system, the traditional static electricity supplies supply chain will be gradually replaced by the highly flexible, data-driven material supply chain. The maximum value of the large data is visual, demand plan and risk management, the application of the large data can make power supplies supply management more effective.

\section{POWER SUPPLIES DEMAND CHARACTERISTICS}

\section{A. Electrical Material Classification Method}

Because of complicated power supplies inventory categories, more varieties, wide coverage, so how much in view of the classification method of materials, several main classification methods are the following:

Divided according to the purchasing mode, can be divided into classes of materials, technology more mature more focused on the standard products, technology, special material and the core of the power system supplies.

Reference to Crutchley supply matrix to scarcity degree and the divisions of the complexity of the market supply, power supplies, leverage can be divided into strategic materials supplies, bottlenecks and general supplies, see Figure 1

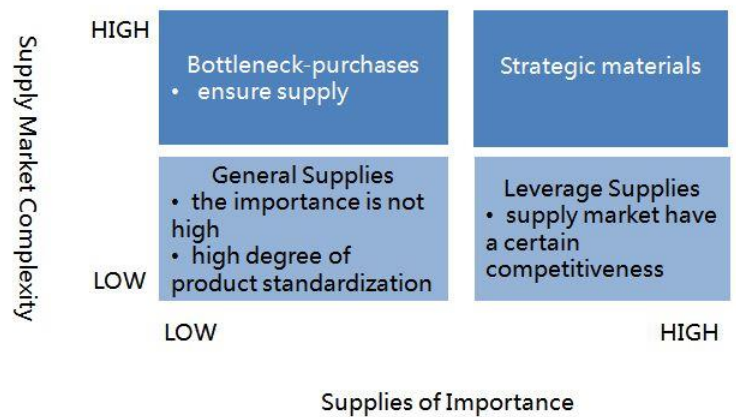

\section{FIGURE I. ELECTRICAL MATERIAL CLASSIFICATION MATRIX}

The classification of Shanghai electric power supplies based on its first ERP material classification, second to meet the functional division by other classification, financial accounting and other business needs. Its purpose is to standardize to the ERP material classification, so very detailed ERP material classification, applicable across the country, as far as possible the unified, standardized and convenient management. This is mainly embodied in its ERP material on the unified national application number.

\section{B. Power Supplies Demand Characteristics}

To research the power supplies demand model, then you must have a deep understanding of demand for electricity supplies characteristics. Specifically, material demand plan of strong and weak law of unstable, different sizes and updating speed, volatility. In order to more accurately describe the power supplies demand characteristics, in this paper, it can be divided into the following nine categories:

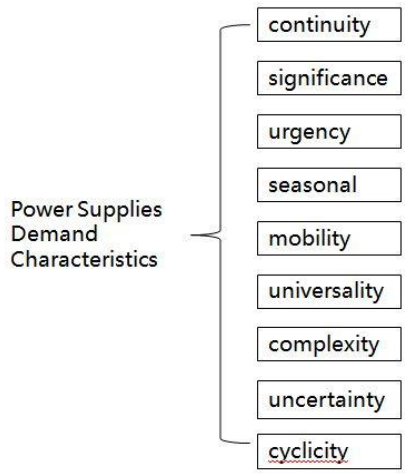

FIGURE II. POWER SUPPLIES DEMAND FEATURES NINE CLASSES

Continuity: ensure the uninterrupted power supply determines the power supplies demand is continuous. 
Importance: related to the operation of the power system. Depends on the material the role of the normal operation of power grids. Such as variable and the shortage of high voltage cable can cause large area blackout for a long time, belong to high importance.

Urgent: grid uninterrupted power supply requirements of the material is must ensure the timely and effective. In the power grid failure should be finished as soon as possible to repair, ensure customer power.

Seasonal: power equipment deployment scattered around, most of the equipment exposed in the open air, vulnerable to natural disasters. And natural disasters are seasonal, so the grid material also has certain seasonal division, caused by different season needs change.

Liquidity: how often the attribute description for material demand. For frequent need of the goods the reserve cost be taken into account when making demand strategy whether to reserve enough, in order to reduce the cost of batch purchase.

Generality: due to the difference of actual situation around, electrical material has the characteristics of differentiation, in order to guarantee the normal response, should consider when reserve generality and universality lower supplies needs more reserves.

Complexity: power equipment widely in spatial distribution and geographical environment. Different regional transportation, distribution, assembling, replacement has different standards, requirements and difficulties, caused the complexity of power supplies.

Uncertainty: uncertainty is one of the important features, power supplies demand in a certain period of time may occur some or several supplies a surge in demand.

Cyclical: materials has a life cycle, part of the equipment need to be replaced regularly, so the material demand is cyclical.

\section{Electrical Material Supply and Demand Characteristics}

For the power supplies of supply and demand characteristic, combined with actual project. Demand for a project characteristic, breakthrough point to analyze material demand response and response time requirements. Supply characteristic, according to the material supply mode and purchasing strategy, analyze the present situation of internal and external supply response will focus on the internal and external response time. Internal supply response time refers to the time of collecting orders combined. External supply response time refers to the supplier delivery cycle.

Combined with the material demand and supply analysis, materials can be divided into the following three categories:

Short supplies: demand period shorter than the shortest supply guide period (i.e., response time), regular purchasing supply cannot meet demand.

Critical supplies: demand period is longer than the shortest lead period, shorter than the longest guide period, regular procurement has a certain probability can't meet the demand.
Abundant supplies: demand period is longer than the longest guide period, regular procurement can meet the demand.

For short board material, the problem in general the uncertainty on the production side, belongs to a production bottleneck.

For critical supplies, problem generally in the uncertainty of demand side and supply side, belong to the type requirements under uncertainty and lack of response.

For abundant materials, basic driven by demand side uncertain problems caused by uncertain supply side.

According to these three kinds of material characteristics, for each process stage of information gathering, sharing and mining processing is essential, so we can timely find problems and solve, can also according to the historical data to forecast the future demand for goods and external supply situation, to optimize the management, improve efficiency.

\section{UNDER THE LARGE DATA OF ELECTRIC POWER SUPPLIES DEMAND MODEL OPTIMIZATION}

Internet of things issued by the ministry of the 12th five-year plan put forward in four key technical innovation projects, respectively: information processing technology, information awareness, information transmission technology, and information security technology. In the information processing technology, mass data storage, data mining, intelligent video image analysis is an important part of the technology of data. The other three key technology innovations are closely related to the big data technology. Generation, data acquisition, storage, processing, conversion, calculation and analysis of the mining process, display and use is the current big data for the entire cycle of data management, which all used a series of process technology and idea is the technology of data.

\section{A. Power Supplies Demand Big Data Platform Architecture}

To optimize material demand patterns of the first step to collect first integration project material purchasing process of various types of data, and inventory management of all kinds of data. In data processing, data is generally divided into structured data and unstructured data. Structured data for characters, namely implementation can be expressed in a two-dimensional table structure to logical data. Unstructured data is cannot use a two-dimensional table structure to express the logic data, including text, images, audio, and video and so on. Traditional data storage method is stored in the database for structured data, unstructured data stored on the local hard disk, we can find that for such storage way for different data we need to adopt a different approach, not only low efficiency and can cause the local data missing. In big data platform, through the establishment of unstructured data center and distributed file system to receive the unstructured data. At the same time, to develop special images, text and audio data processing algorithm, special analysis model, and in a short period of time digging into the valuable information.

Through analyzing the demand features in-depth research, comprehensive build and manage procurement requirements model, including not limited to statistical analysis model, forecast analysis model and analysis model of knowledge mining. 
In terms of the results show, big data platform has the function of report automatically generated, including set theme analysis report and instant custom analysis report.

In the big data platform to have multiple function analysis scenarios and custom scene, this part is called analysis of subject library, including set theme analysis, real-time analysis theme application and unstructured data analysis applications.

In the whole framework of big data, analysis of data mining platform in the core position, connect down all kinds of data sources, up for the daily report, statistical analysis, real-time monitoring and trend prediction statements show business analysis and application, data analysis, data calculation, etc.
Big data analysis mining platform by integrating a variety of sources of structured, semi-structured, and unstructured data, provide a common computing framework and method, for the upper application based on large data analysis and decision support tools and application development services ability, at the same time provide data model, data management, system management, and other auxiliary management, configuration tools. Big data analysis functions to meet the demand of based on the characteristics of mining platform and mode of procurement phase of the data analysis of three of the business needs of the application direction, namely online analytical applications, data mining application and unstructured data analysis applications.

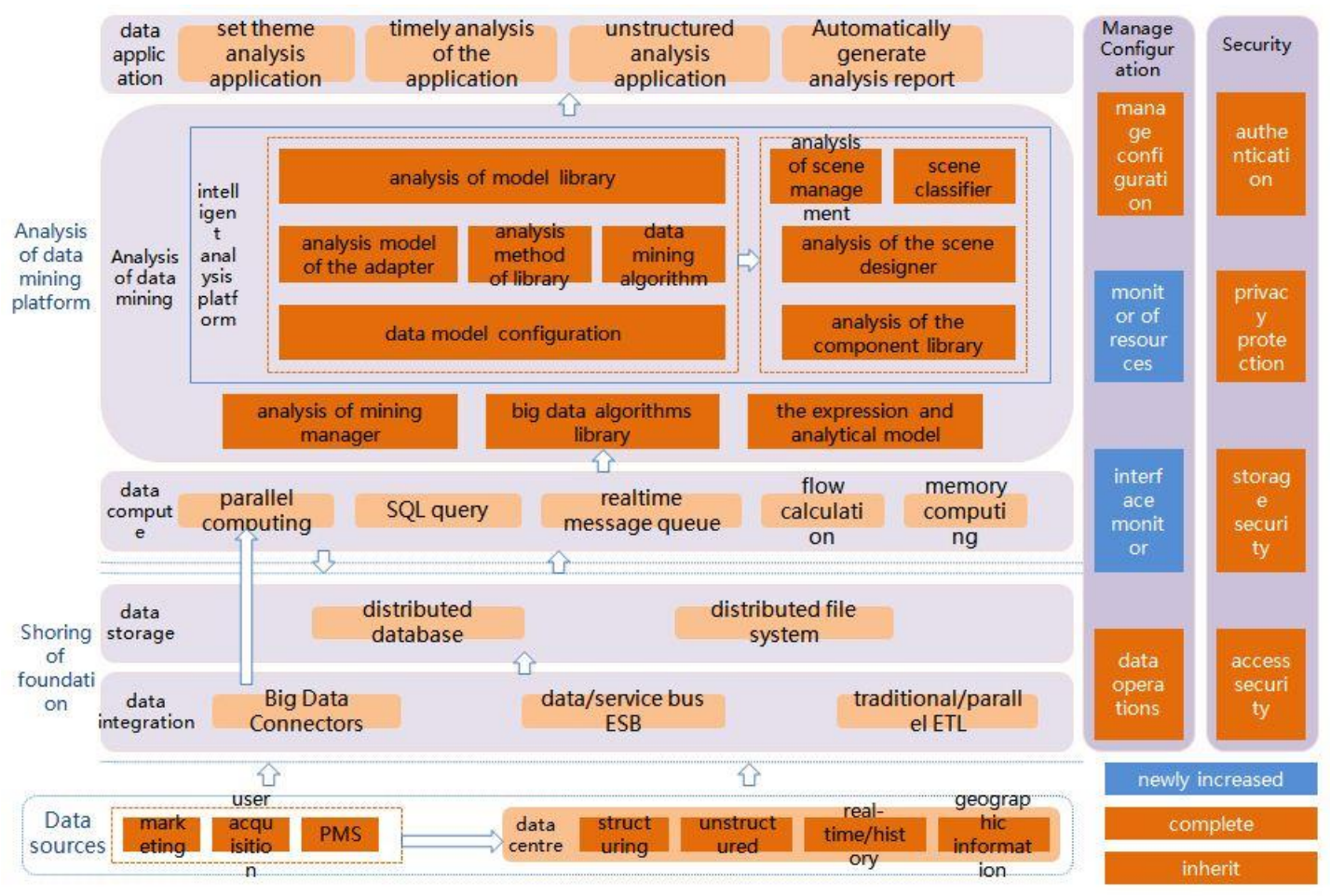

FIGURE III. POWER SUPPLIES LARGE DATA MINING PLATFORM ARCHITECTURE

\section{B. Power Supplies Demand Big Data Function Design}

For data mining, instant online and unstructured data analysis three main application direction of business requirements, large power supplies demand data function design has the following four contents:

Data access: provide the demand information of data access and clean function. Data in information system data access, external data import and import grid topology as the main access to the source.

Analysis model of management: the analysis model of procurement requirements of construction and management of all, including statistical analysis model, forecast analysis model and analysis model of knowledge mining.

Automatically generate analysis report: the link is responsible for the analysis of generation and display, including the established model set theme analysis report, instant give reference and analysis report of visual display.
Phase analysis subject library management: provide procurement guide analysis scene management and display platform, including set theme analysis applications, real-time analysis and unstructured data analysis application.

\section{Power Supplies Demand Forecasting Based On Big Data}

Based on big data platform, data efficiently use, according to different materials demand forecasting can quickly enable different demand forecasting model, to achieve relative to predict accurately and rapidly the requirements.

The choice of the model in two steps: the first step, according to characteristics of materials classification, characteristics and demand model match, find out the model for the classification, basic scope of model selection. The second step, how to in several optional model to determine the optimal method of the model.

Determine the scope of the model of each type of material can be according to the characteristics of the material classification, choose several kinds of model suitable for this 
kind of material. Because the use of big data analysis platform, for the procurement, screening will demand forecasting model, based on historical data and forecast data deviation, find out the minimum error of the model as the best model.

\section{SUMMARY}

In today's project supplies demand more and more complex, supplies demand, supply timely response so as to ensure the normal continuity for the project schedule, reduce unnecessary costs, the traditional method in dealing with such matters has been compromised. Through data mining platform construction, large data mining analysis method was applied to material demand forecast. Through data mining prediction results, adjust the procurement mode, prepared in advance, to ensure that the material demand side and supply side can response in time, so as to optimize the power supplies demand patterns.

\section{REFERENCES}

[1] Cankui Yang. The Whole Process of Supply Chain Management Decision Analysis System Based on The Large Data Exploration [J]. Mechanical and Ele,2016, (36):138-139.

[2] Use Big Data to Predict Customer Demand [N]. Jielin Luo. Shenzhen Economic Daily. 2015-07-10 (A04).

[3] Exploring The Demand Frecast on The Basis of Large Data[N]. Chuanzhao Shou. Oriental tobacco. 2014-03-03 (003).

[4] Feijuan Li. Application Rsearch On Model Building and The Internet of Things [D]. North China Electric Power University (Beijing),2016.

[5] Jian Hog, Jinyu Chen. Supplies All The Supply Chain Data Application Research [J]. Logistics Engineering And Management,2017, (03):65-67.

[6] Under The Background of Big Data and Application Research On Electric Power Industry Data [J]. Chao Chen, Shunshi Zhang, Shouwei Shang, Fei Sun. Modern Electronic Technology. 2013(24). 